\title{
Three steps to a healthy mouth
}

\author{
This article presents the evidence supporting the use of a three-step home care regimen to \\ maintain good oral health. Brought to you by Johnson \& Johnson, the makers of LISTERINE ${ }^{\circledast}$
}

We know that twice-daily brushing and interdental cleaning are essential in achieving and maintaining oral hygiene. ${ }^{1}$ They displace and dislodge plaque bacteria that can cause gingivitis and periodontal disease from the tooth surface. ${ }^{2}$

However, we also know that, for a number of reasons, brushing and interdental cleaning are insufficient for the majority of the population to eliminate plaque bioflim. ${ }^{1,3}$ This is supported by the data published in the most recent Adult Dental Health Survey (2009); despite 75\% of dentate adults surveyed claiming to brush their teeth at least twice a day, $66 \%$ had visible plaque. ${ }^{3}$

All of this suggests that there remains an unmet need when it comes to implementing an effective home care regimen between check-up and hygiene appointments.

\section{An adjunctive third step}

Barnett (2006) suggested that this gap in preventive care provides: ' $[\ldots]$ a clear rationale for incorporating effective antimicrobial measures, such as use of an antimicrobial mouthrinse, into daily oral hygiene regimens.'2

Looking at the issue from both individual health and general public health perspectives, he also considered that using an antimicrobial mouthrinse on a daily basis would have a significant effect on plaque control, making it a cost-effective and significant adjunct to mechanical cleaning. ${ }^{2}$

Building on this, in 2014 Charles and colleagues sought to determine the ability to achieve gingival health in the short-term through daily rinsing with an essential oil-containing antimicrobial mouthrinse. ${ }^{4}$

They concluded: 'Significantly more healthy gingival sites and virtually plaque free tooth surfaces can be achieved as early as 4 weeks with use of an essential oil antimicrobial mouthrinse. This finding continues through 6 months twice daily use as part of oral care practices compared to mechanical oral hygiene alone."

This is supported by Boyle and colleagues (2014), who explored the differences between a variety of mouthrinses on common oral conditions. ${ }^{5}$ They came to the conclusion that when a mouthwash is used for fewer than three months, those containing chlorhexidine are the most effective of the preparations they considered. ${ }^{5}$ However, when used for six months or longer, essential oil mouthwashes were shown to equal or exceed the effect of chlorhexidine in controlling plaque as an adjunct to standard care. ${ }^{5}$

In 2015, Chapple and colleagues reported on the consensus views of Working Group 2 of the 11th European Workshop in Periodontology. ${ }^{6}$ They related that:

- 'There is a universal recommendation to brush twice daily for at least 2 minutes with a fluoridated dentifrice. For periodontitis patients, 2 minutes is likely to be insufficient.'

- 'Daily interdental cleaning is strongly recommended to reduce plaque and gingival inflammation.'

- 'In patients with gingivitis, the adjunctive use of chemical agents for plaque control offers advantages.'
They further concluded that, '... where improvements in plaque control are required, adjunctive use of antiplaque chemical agents may be considered. In this scenario, mouth rinses may offer greater efficacy but require an additional action to the mechanical oral hygiene regime.'

Furthering the supporting evidence is Araujo and colleagues' (2015) meta-analysis. ${ }^{7}$ They were able to show the oral health benefits of using an essential oil-containing mouthrinse as an adjunct to mechanical cleaning. ${ }^{7}$

Responder curves ${ }^{*}$ plotted by the authors show that a mean average of $36.9 \%$ subjects using mechanical methods with an essential oil mouthrinses experienced at least $50 \%$ plaque-free sites after 6 months compared to just $5.5 \%$ of patients using mechanical methods alone. ${ }^{7}$

Summarising the results, Araujo and colleagues (2015) were able to provide strong evidence that there are statistically significant greater odds of patients achieving a '...cleaner [...] mouth, which may lead to prevention of disease progression...' if patients add an essential oil mouthrinse to their daily mechanical cleaning regimen at home. ${ }^{7}$

\section{Accessing preventive care}

In terms of preventive care, it has been put forward that dental plaque, the main cause of oral disease, can be removed through the mechanical means of brushing and interdental cleaning. ${ }^{5}$ However, it has also been suggested that a significant amount of plaque remains on the teeth even following the implementation of such a regimen, while the soft tissues remain largely untouched. ${ }^{5}$

Since mechanical cleaning alone is therefore insufficient, it has been suggested that a chemotherapeutic mouthrinse - such as one containing essential oils - offers a considerable advantage when used as an adjunct to mechanical cleaning, as it, '...can reach virtually all residual plaque.....

Indeed, the hydrodynamic ability of a mouthwash allows it to reach all five exposed tooth surfaces, so, given the scientific evidence supporting its efficacy, for most patients it is a logical long-term adjunct to a daily preventive regimen.

References

1. Sharma $\mathrm{N}$ et al. Adjunctive benefit of essential oil-containing mouthrinse in reducing plaque and gingivitis in patients who brush and floss regularly: a six-month study. J Am Dent Assoc. 2004; 135: 496-504.

2. Barnett M L. The rationale for the daily use of an antimicrobial mouthrinse. JADA 2006 ; 137: $165-21 S$.

3. Adult Dental Health Survey 2009. The Health and Social Care Information Centre 2011.

4. Charles C A et al. Early benefits with daily rinsing on gingival health improvements with an essential oil mouthrinse - post-hoc analysis of 5 clinical trials. Journal of Dental Hygiene 2014; 88 (Suppl.): 40-50.

5. Boyle et al. Mouthwash use and the prevention of plaque, gingivitis and caries. Head \& Neck Oral Diseases 2014; 20: 1-76.

6. Chapple I L C et al. Primary prevention of periodontitis: managing gingivitis. J Clin Periodontol 2015; 42 (Suppl. 16): S71-S76.

7 Araujo M W B et al. Meta-analysis of the effect of an essential oil-containing mouthrinse on gingivitis and plaque. JADA 2015; 146: 610-622.

* Responder curves plot the proportion of participants within each treatment group achieving at least the given percentage of healthy sites, for all possible percentages of healthy sites $(0-100 \%)$ ? 